\title{
A true science of consciousness explains phenomenology: comment on Cohen and Dennett
}

\author{
Johannes J. Fahrenfort ${ }^{1,2}$ and Victor A.F. Lamme ${ }^{1,2}$ \\ ${ }^{1}$ Brain \& Cognition, Department of Psychology, University of Amsterdam, Weesperplein 4, 1018 XA Amsterdam, The Netherlands \\ ${ }^{2}$ Cognitive Science Center Amsterdam (CSCA), University of Amsterdam, Plantage Muidergracht 22-24, 1018 TV Amsterdam, \\ The Netherlands
}

In their recent article in TiCS [1], Cohen and Dennett propose that consciousness is inextricably tied to one's ability to report about the contents of experience (an 'access-only' theory). They contrast this with theories that distinguish mechanisms that create the contents of experience from mechanisms that allow one to report about this content ('phenomenal-access' theories). These they claim to be unfalsifiable, and therefore beyond the realm of science. We argue below that this assertion is wrong and based on a misguided belief about what a theory of consciousness needs to explain.

Cohen and Dennett maintain that phenomenal-access theories are 'dissociative', as though these theories propose mechanisms of consciousness that are devoid of function. They seem to suggest that access itself is the function that needs to be explained. Hence, in their description of a 'perfect experiment', access is the only test of whether something is experienced. However, their setup does not clarify how the contents of experience come about, and therefore by its very nature has no explanatory power. A real perfect experiment would provide the neural mechanisms that explain functional properties of consciousness (Figure 1).

Such mechanisms should be able to integrate contextual information across the visual field, making inferences about its input while resolving perceptual ambiguity. They should be able to dynamically group image elements together, creating perceptual unity and perceptual organization. It is well established that object features are represented by neurons with receptive fields that are too small to achieve such integration. So how could a distributed network such as the brain achieve this? The theory of

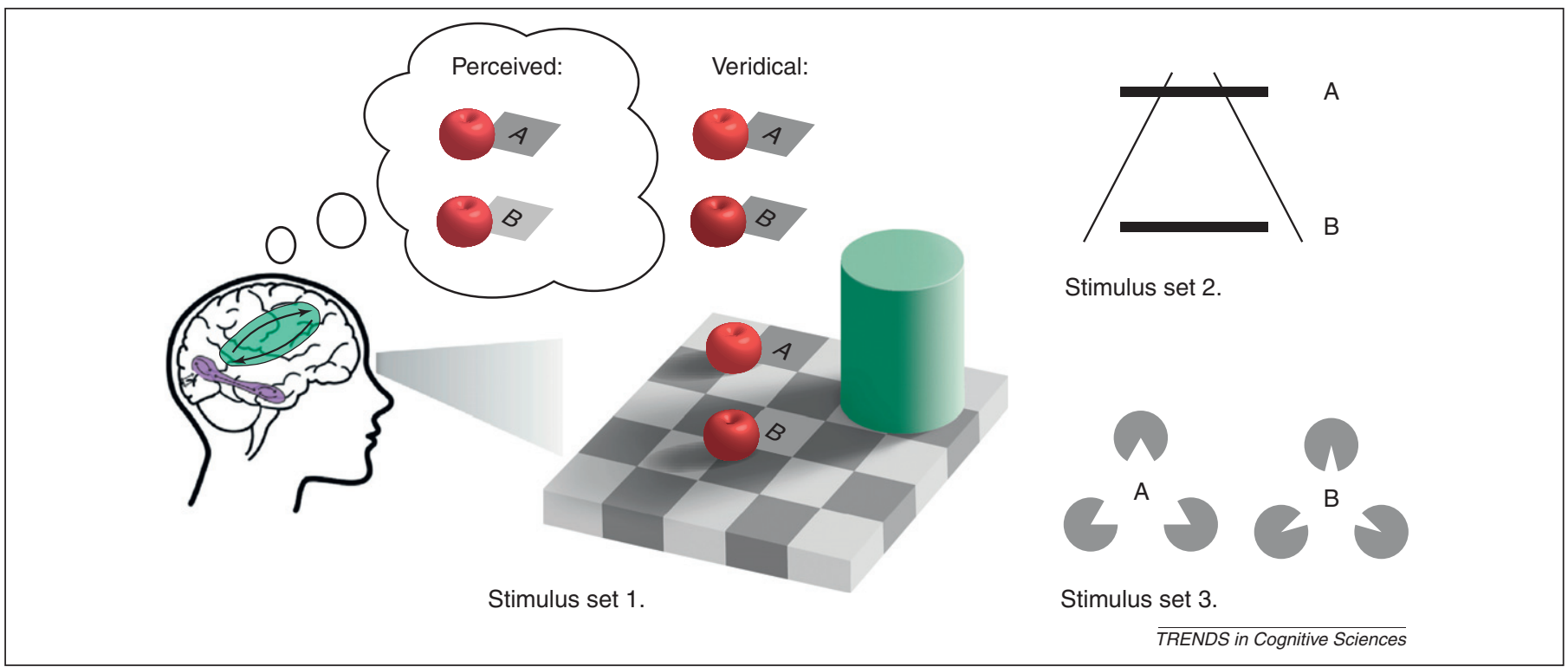

Figure 1. The perfect experiment. During the extended checkerboard shadow illusion (stimulus set 1), the perceived color of surfaces and objects are dependent on the

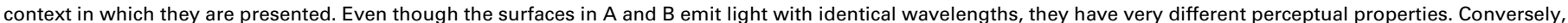
the apples seem to have the same color, whereas in fact apple B is much darker than apple A. During the Ponzo illusion (stimulus set 2), the bar in condition A is perceived to be longer than the bar in condition B, whereas in fact they are equal in size. During the Kanisza illusion (stimulus set 3 ), condition $A$ induces the perception of a triangle lying on top of the inducers, whereas condition B lacks such surface perception. If, under a wide range of circumstances, one can establish neural mechanisms that are consistently tied to the perceptual (as opposed to the veridical) state of visual input, while in addition having functional properties that explain these states, then by current scientific standards, one may infer that these mechanisms are involved in generating the contents of experience. If these putative mechanisms (purple) remain when the neural mechanisms of access (green) are removed, one should conclude that consciousness without access exists. If consciousness critically depends on access, the hypothesis that perception without access exists is falsified.

Corresponding author: Fahrenfort, J.J. (j.j.fahrenfort@uva.nl); Lamme, V.A.F. (v.a.f.lamme@uva.nl) 
local recurrency argues that neurons with receptive fields large enough to encapsulate entire objects, bind together features through recurrent interactions, subserving image segmentation and perceptual organization [2]. In the theory of coalitions of neurons [3], neurons engage in the formation of coalitions that represent unified percepts of otherwise distributed information. The functional property that these theories have in common can be loosely summed up as the ability to integrate or bind information across spatially separated sets of neurons to infer perceptual rather than physical attributes of visual stimuli [4,5]. Phenomenal-access theories propose that this property explains key elements of conscious experience, as many observations show that it is characteristic of the emergence of phenomenology [2,6]. Importantly, perceptual organization does not require selective attention [6,7], but rather serves as input for it $[8,9]$. Thus, although access-only theories allege that representations are only phenomenal when reported, access itself does not seem to be involved in generating the contents of experience, and therefore it has little power to explain phenomenology [10].

Now if it turns out that the neural mechanisms of perception established in our perfect experiment subside when their contents cannot be accessed- as when the green connections in Figure 1 are lesioned out as Cohen and Dennett propose- the idea of phenomenology without access would be falsified. In that case, and only then, access would need to be incorporated into theories of phenomenology. If however, given the stimuli presented in Figure 1, these neural mechanisms continue signaling the perceptual states corresponding to condition A and condition B, even though subjects are not able to report about them, the parsimonious account is to infer that perceptual states continue to exist without access. According to Cohen and Dennett however, these mechanisms can no longer be trusted to operate as previously established, only because the subject has lost his or her ability to report on them. If anything, rather than disproving the scientific validity of phenomenal-access theories, this step places 'access-only' theories outside the realm of science.

\section{References}

1 Cohen, M.A. and Dennett, D.C. (2011) Consciousness cannot be separated from function. Trends Cogn. Sci. 15, 358-364

2 Roelfsema, P.R. (2006) Cortical algorithms for perceptual grouping. Annu. Rev. Neurosci. 29, 203-227

3 Crick, F. and Koch, C. (2003) A framework for consciousness. Nat. Neurosci. 6, 119-126

4 Singer, W. and Gray, C.M. (1995) Visual feature integration and the Temporal Correlation Hypothesis. Annu. Rev. Neurosci. 18, 555586

5 Tononi, G. and Edelman, G.M. (1998) Consciousness and complexity. Science 282, 1846-1851

6 Super, H. et al. (2001) Two distinct modes of sensory processing observed in monkey primary visual cortex (V1). Nat. Neurosci. 4, 304-310

7 Scholte, H.S. et al. (2006) The influence of inattention on the neural correlates of scene segmentation. Brain. Res. 1076, 106-115

8 Qiu, F.T.T. et al. (2007) Figure-ground mechanisms provide structure for selective attention. Nat. Neurosci. 10, 1492-1499

9 Roelfsema, P.R. et al. (2007) Different processing phases for features, figures, and selective attention in the primary visual cortex. Neuron 56 , 785-792

10 Lamme, V.A.F. (2010) How neuroscience will change our view on consciousness. Cogn. Neurosci. 1, 204-220

\title{
Response to Fahrenfort and Lamme: defining reportability, accessibility and sufficiency in conscious awareness
}

\section{Michael A. Cohen ${ }^{1}$ and Daniel C. Dennett ${ }^{2}$}

\author{
${ }^{1}$ Vision Sciences Laboratory, Department of Psychology, William James Hall, Harvard University, Cambridge, MA 02138, USA \\ ${ }^{2}$ Center for Cognitive Studies, Department of Philosophy, Tufts University, Medford, MA 02155, USA
}

In their letter to TiCS [1], Fahrenfort and Lamme (F\&L) bring up two issues in response to our position [2] that we address here.

The first issue concerns the relationship between access and reportability. F\&L write that we 'propose that consciousness is inextricably tied to one's ability to report about the contents of an experience'. Their criticism seems to rest on the belief that we claim that consciousness is tied to the ability to do things such as talk or press a button. This is not our view. If it were, it would clearly be wrong: information can be conscious yet verbally unreportable. Some patients with locked-in syndrome or who are in a persistent vegeta-

Corresponding author: Cohen, M.A. (michaelthecohen@gmail.com); Dennett, D.C. (daniel.dennett@tufts.edu). tive state have been identified as conscious even though they cannot talk about their experiences. However, it must be stressed that these patients do still report their experiences by engaging in mental-imagery tasks during functional magnetic resonance imaging (fMRI) and it is these 'reports' that allow clinicians to identify these patients as conscious [3-5]. What enables the willful modulation of mental imagery? The mechanisms of access: attention, memory, decision-making, and so on. Without these mechanisms, a patient could not hold the instructions in memory, attend to the words being spoken over the sounds of the scanner and decide to imagine the stimuli that correspond with a predesignated answer. Without these mechanisms, there would be no reason to believe that these patients are conscious of anything at all. 